\section{Minga virtual: herramientas de co-creación mediadas por las TIC}

Paola Banderas Quirola ${ }^{(1)}$

Resumen: El año 2020 enfrentó la pandemia SARS-COV2, que trajo consigo la virtualización de la educación a nivel mundial y con ella nuevos retos en las estrategias de enseñanza-aprendizaje. Este salto fue un gran paso para la educación donde el éxito del proceso responde al compromiso de los actores para con la cátedra y la modalidad. Un ejemplo de este proceso ha sido el Taller de Diseño Participativo, asignatura que pertenece a la sección formativa del rediseño de la Carrera de Productos de la PUCE y tiene la particularidad de desarrollar métodos de co-creación con comunidades vulnerables que tienen poco acceso al diseño y las ventajas que proporciona. En el segundo semestre del año 2020 esta asignatura fue impartida por primera vez y desarrollada en colaboración con la comunidad Sara Sisa. Este grupo de mujeres indígenas de Llano Grande está recuperando sus costumbres a través del bordado y han encontrado en esta técnica una fuente de ingreso económico, satisfacción personal, empoderamiento y desarrollo colectivo.

El presente artículo expone herramientas que permiten acercar la academia a la realidad local mediante la minga virtual como proceso cooperativo para la construcción de proyectos que tomen en cuenta las diferencias como fortalezas para la construcción de productos innovadores con valor. Cabe resaltar que la institución con su bagaje de formación de calidad ha desarrollado varios medios de comunicación virtual entre docentes y estudiantes, lo que ha permitido formar desde varias aristas, profesionales capacitados, empáticos y comprometidos con la sociedad, demostrando que los procesos de co-creación pueden darse con éxito apoyados en las nuevas tecnologías de la información y la comunicación.

Palabras clave: Diseño participativo - educación virtual - TIC - proyectos colaborativos - co-creación.

[Resúmenes en inglés y portugués en las páginas 53-54]

(1) Paola Banderas Quirola. Adscrita a la Pontificia Universidad Católica del Ecuador, en la Carrera de Diseño de Productos de la Facultad de Arquitectura, Diseño y Artes. Diseñadora por la Pontificia Universidad Católica del Ecuador (PUCE) y Máster en Desarrollo de Proyectos de Innovación y Producto por la Universidad de León (España). Docente a tiempo parcial en la Carrera de Diseño de la PUCE. Investigación en Diseño con identidad. Diseño Estratégico. Diseño Participativo. Diseño textil. Código ORCID: 0000-00022073-7611 


\section{Introducción}

El ser humano por su naturaleza es un ser social, y es mediante la relación con el contexto y otros seres donde va conformando su identidad. Es así como, para la construcción del "yo" o la personalidad, se conjuga tanto la biología del individuo, así como las experiencias de vida y factores del entorno como costumbres, tradiciones, prácticas sociales, símbolos entre otros.

Ese contexto en el cual se encuentra inmerso es una fuente constante de información que permite un aprendizaje basado en intercambio multidireccional donde el ser es afectado por todo lo que lo rodea y, a su vez, él impacta a su entorno. Es decir, dicha relación incide en ambos actores: en el ser individual y en el colectivo social. Es una simbiosis, que va transformando lentamente la cultura, la misma que constituye un componente fundamental del proceso de adquisición de conocimiento y por ende de la educación en un entorno. Como se menciona:

Todos los hombres pertenecientes a una determinada sociedad son actores y autores de la cultura que produce esa sociedad, son elementos de "un mismo clima cultural", entendiéndose por tal no la uniformidad ni la pasividad, sino todo lo contrario: la lucha entre corrientes culturales diversas y opuestas y su necesaria unidad, como base del movimiento cultural en su conjunto. Ron (1977, p.32)

Ese contexto es complejo y se conforma por distintas realidades, el acercarse a ellas, permite comprenderlas, enriquecer la mirada desde otras perspectivas y promueve la empatía. Capacidades que se deben fomentar desde la academia, dada la importancia de dichas habilidades en los perfiles profesionales requeridos en la actualidad.

Por ello, la cátedra de Taller de Diseño Participativo, busca un acercamiento del estudiante a las diferentes comunidades, asociaciones o colectivos del Ecuador para, desde una vinculación de la academia con los entes sociales, impulsar al desarrollo y empoderamiento de grupos vulnerables. Así, desde la formación de los jóvenes entorno a la disciplina del diseño, se desarrollan proyectos cooperativos en base a la realidad de una comunidad, identificando su naturaleza, recursos y expectativas que, mediante el intercambio de saberes y la aplicación de herramientas y técnicas de la metodología participativa, permiten consolidar las competencias de formación específica de los estudiantes de la carrera de diseño de productos de la PUCE.

Este enfoque de co-creación, no es nuevo. Viene inmerso en la cosmovisión andina y se materializa en la MINGA, entendida por Borja como: "una milenaria institución de los grupos indígenas de los andes que consiste en la reciproca prestación de servicios gratuitos" (2012, p. 650) y definida por Cruz, M. como: "la idea y apreciación del mundo de las personas, tras la cual contemplan y revelan su entorno natural y cultural, es el producto de un proceso de evolución del pensamiento." (2018, p.123).

La minga entendida desde sus dos acepciones "Reunión de amigos y vecinos para hacer un trabajo gratuito en común y Trabajo agrícola colectivo y gratuito con fines de utilidad social" (Real Academia Española, s.f., definición 1-2), devenga en un ideal de trabajo colaborativo en la búsqueda del bien común, y se contrapone a la instaurada práctica de 
crecimiento personal como único fin de desarrollo. Este ideal de trabajo cooperativo se traspasa al proceso de enseñanza aprendizaje, dónde cada uno de los actores, sean estudiantes, artesanos, empresarios, trabajadores, docentes, o de cualquier otra índole, pasan a ser implicados, actores o participantes que asumen un rol activo en la construcción cooperativa de una meta.

Partiendo desde la cosmovisión andina dónde la suma de saberes se potencia, cada participante es único y puede aportar en igual medida, y donde el bien común sobrepasa al bien individual porque es la conclusión de un crecimiento personal a través de un crecimiento colectivo. Este rol implica un compromiso para y con el equipo, dónde el éxito y el resultado del proyecto es proporcional a la entrega de cada integrante, la planificación adecuada, la fluida coordinación entre los miembros del equipo y la comunicación y socialización oportuna del trabajo.

Con ello no se pretende romper la individualidad, cabe aclarar el respeto por el individuo como la base de desarrollo personal que es irrefutable. Como plantea Vargas Llosa citado en Castro "el individuo no debe vender sus fines, sueños o propósitos a un colectivo, aunque se sienta parte de él" (2019, p.147). Para compresión plena del artículo, se pretende reflexionar sobre las ventajas que conlleva el trabajo cooperativo como búsqueda de un desarrollo conjunto en el que los individuos se potencian en la interacción de saberes que a su vez permiten impulsar la individualidad y promover el bien común, todo esto impulsado desde las tecnologías de la información y la comunicación (TIC). Es un aprendizaje dialógico que como afirma Aubert et al. (2008) "se produce en interacciones que aumentan el aprendizaje instrumental, favorecen la creación de sentido personal y social, están guiadas por principios solidarios y en las que la igualdad y la diferencia son valores compatibles y mutuamente enriquecedores". p.167).

Idiosincrasias, perspectivas y tecnologías bajo las cuales se desarrolla el ejercicio académico del Taller de Diseño Participativo de la Pontificia Universidad Católica del Ecuador, que se vincula a comunidades ${ }^{1}$ para desde la relación academia sociedad poder enriquecer el proceso de enseñanza aprendizaje desde las metodologías participativas, aportar al desarrollo de la realidad del país mediante el empoderamiento y acercamiento de grupos vulnerables a los beneficios que conlleva la aplicación del diseño como herramienta creativa para solucionar problemas, encontrar oportunidades, definir estrategias que permiten innovar en medios globales (generales) y locales (contextualizados).

\section{La co-creación}

La co-creación definida por la Real Academia de la Lengua Española como: co-coetáneo, cooperar, colateral, corresponsable (Real Academia Española, s.f., definición 1) y creación: f. Acción y efecto de crear; (Real Academia Española, s.f., definición 1), es decir en conjunto: acción o efecto de crear cooperativamente; al ser aplicada como una metodología constituye una manera de alcanzar uno o varios objetivos y entendida como estrategia permite crear experiencias valiosas con clientes, usuarios, trabajadores y demás participantes. En el taller de diseño Participativo se la aplica como un proceso mediante el cual se logra vincular varios actores de distintos contextos para potenciar sus conocimientos. 
La co-creación se ha venido empleando desde siempre, pues se fundamenta en la colaboración que es una característica propia del ser humano como ser social. Definida por Wilches (2019, p.85) como "un proceso colaborativo para crear valor que cuenta con la participación de actores internos y externos, con el propósito de obtener beneficios en común", se ha venido empleando las últimas décadas en varios ámbitos como el social, comercial o académico.

Dependiendo del contexto en el que se desarrolle y el enfoque del proyecto, se verán implicados distintos actores, que pueden ser empresarios, emprendedores, ministerios, ONG, profesionales, académicos, estudiantes, comuneros, ciudadanos, en fin, cualquier persona ligada al proyecto que pretenda aportar desde su identidad, bagaje de conocimientos y predisposición para con la meta del proyecto pues lo que impera en la actividad es la colaboración.

\begin{tabular}{ll}
\hline \multicolumn{1}{c}{ Autores } & \multicolumn{1}{c}{ Definición } \\
\hline Herrera-González e Hidalgo-Nuchera (2018) & $\begin{array}{l}\text { Mecanismo de agregación de valor en donde se involucran diferentes actores, siendo los clien- } \\
\text { tes los que participan activamente en las etapas de ideación durante el proceso de innovación. }\end{array}$ \\
\hline Agrawal y Rahman (2019) & Actividades colaborativas y la creación de valor para los actores involucrados en el proceso. \\
\hline Saunila, Ukko y Rantala (2019) & $\begin{array}{l}\text { Cultura de apertura asociada a la vinculación de clientes y procesos de cambio para la crea- } \\
\text { ción de valor. }\end{array}$ \\
\hline $\begin{array}{l}\text { Rosales-Chávez, Cruz-López, Domínguez- } \\
\text { Arce y Parra-Castrillón (2019) }\end{array}$ & Actividades que conducen a la obtención colaborativa y evolutiva del valor. \\
\hline Grönroos \& Voima (2013) & $\begin{array}{l}\text { Creación de valor durante el uso, resultado del intercambio recíproco de intereses de los clien- } \\
\text { tes y las organizaciones. }\end{array}$ \\
\hline Roser, Defillippi y Samson (2013) & $\begin{array}{l}\text { Proceso interactivo, creativo y social entre las partes interesadas, que inicia por las organiza- } \\
\text { ciones con el objetivo de crear valor. }\end{array}$ \\
\hline Ind y Coates (2013) & $\begin{array}{l}\text { Proceso creativo de interacción simultánea entre las necesidades de los interesados y la orga- } \\
\text { nización, con el objetivo de generar una nueva idea. }\end{array}$ \\
\hline Lambert y Enz (2012) & $\begin{array}{l}\text { La creación conjunta de valor que abarca un ciclo de tres fases en las que interactúan clientes } \\
\text { y proveedores: 1) elaboración conjunta de propuestas de valor, 2) determinación de la actuali- } \\
\text { zación de valor y 3) determinación de valor. }\end{array}$ \\
\hline Grönroos (2012) & $\begin{array}{l}\text { Actividad de colaboración conjunta de valor mediante interacción directa de las partes invo- } \\
\text { lucradas. }\end{array}$ \\
\hline Gummesson y Mele (2010) & $\begin{array}{l}\text { "Centralidad equilibrada" en donde una red de actores intercambia recursos y necesidades, } \\
\text { para poder crear valor y obtener beneficios conjuntamente. }\end{array}$ \\
\hline
\end{tabular}

Figura 1. Definiciones agrupadas según Wilches (2019, p.85). Fuente: Wilches, 2019.

A partir de las definiciones anteriores, se encuentran cuatro puntos fundamentales para llevar a cabo el proceso de co-creación: los implicados, el proceso en sí de crear de forma cooperativa, el valor y el resultado. Todos son necesarios, se interrelacionan y en todos los casos unos son productos de otros. Como podemos apreciar en la figura 2, los implicados se interrelacionan en el proceso del cual surge el valor del producto (entendido como la meta final, no como el objeto de diseño), este valor llevado al contexto y utilizado por los usuarios finales, da como resultado el impacto en los implicados y en el entorno. 


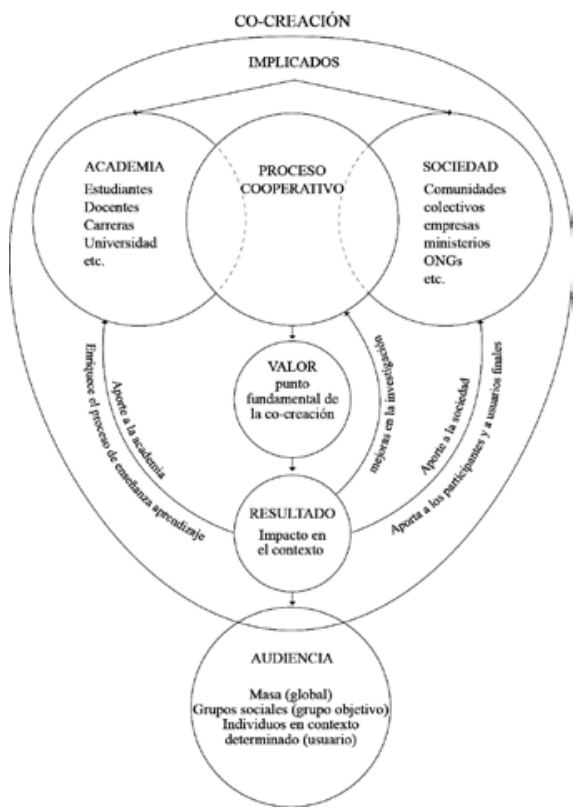

Figura 2. Relación de implicados en la co-creación. Nota: El gráfico expone la relación de implicados mediante proceso cooperativo, de dónde surge el valor y el resultado como puntos fundamentales para la co-creación tomando en cuenta la percepción de la audiencia.

Como se presenta en la gráfica, el proceso que se da entre los implicados (sean éstos de cualquier contexto) da como resultado el punto fundamental de la actividad de intercambio de conocimiento, saberes y experiencias que es el valor aplicado al producto. Dicho valor se establece en torno a la organización con la que se desarrolla el proyecto y con los agentes que participen en el intercambio, tomando en cuenta la percepción de la audiencia. Es la definición de las fortalezas, oportunidades y la diferenciación con la que se puede dotar al fruto resultante de la co-creación enfocado en las necesidades y/o expectativas del usuario, grupo objetivo o quien fuese su receptor. Definido por García (1998, p.1) como "aquello que hace a una cosa digna de ser apreciada, deseada y buscada; son, por tanto, ideales que siempre hacen referencia al ser humano y que éste tiende a convertir en realidades o existencias"; realidades que Windelband llama "el tercer reino" en el que confluye la realidad como experiencia sensible y la esfera de los valores. Todo ello se suscita dentro de la cultura, a lo que Rickert llamó el espacio de las "formaciones de sentido". (Sánchez, 2005, p.3)

Si bien en el caso de la asignatura de Taller de Diseño Participativo, los actores provienen de la academia (estudiantes y docentes del quinto nivel de la carrera de productos) y de la comunidad (artesanos que van vinculándose cada semestre del cronograma académico y con miras de una relación prolongada), en varios casos también se vinculan profesionales de áreas relacionadas a la temática del proyecto, desarrollando en el estudiante capacidades para el trabajo interdisciplinar. Es un ejercicio realizado con las personas y para las 
personas que, como resultado de un proceso académico ligado a la realidad local, genera tres niveles importantes dentro del proceso de enseñanza aprendizaje.

Por un lado, el aporte a la academia. Promueve una formación profunda que nace de la vivencia del estudiante sin que sea el tutor quien se lo transmita. Es un enfrentamiento a realidades diversas en contextos determinados que, mediante el descubrimiento del otro, la generación de confianza, el diálogo contante y la entrega profunda, promueve prácticas educativas inclusivas, empáticas y conscientes de las diversas culturas, identidades y saberes, afectando al discente no solo en su intelecto sino también en su emocionalidad generando reflexión sobre el sentido de la profesión. Adicionalmente, visualizando a la co-creación y al Diseño Participativo como estrategias metodológicas capaces de ser aplicadas en la práctica docente independientemente de la modalidad en la que se aplique (virtual o presencial)

En segunda instancia, genera un aporte relevante a la sociedad. Inicialmente con los artesanos con quienes se desarrolla el proyecto, acercando a la praxis de la comunidad, las ventajas de innovación que aporta la disciplina del diseño. E indirectamente a los usuarios finales, consumidores de los productos realizados por la comunidad, que se ven beneficiados en sus actividades cotidianas vinculadas a los productos de consumo. Aquí vale resaltar que en algunos casos son los artesanos los mismos usuarios finales. Por ejemplo, cuando se realiza un producto centrado en el mejoramiento del proceso ligado a su práctica artesanal.

En tercer lugar, tenemos la mejora en el área de la investigación. El ejercicio académico aporta al avance de la metodología en sí misma, creando nuevas herramientas para su puesta en marcha, adaptando las herramientas existentes para que sean contextualizadas, utilizando recursos del entorno cercano para poder hacerlas accesibles a todos los participantes y/o, la adaptación a distintas necesidades como su aplicación en la modalidad virtual dada la situación actual de distanciamiento social y por concerniente la virtualización de la educación.

\section{La co-creación en la presencialidad y virtualidad}

La co-creación como estrategia de trabajo cooperativo se ha aplicado en mayor proporción como metodología empresarial más que de aprendizaje. Comúnmente se la ha llevado a cabo en la modalidad presencial por su carácter vinculante ya que constituye la manera natural de relacionarse del ser humano. Ésta forma de interacción cambia drásticamente después de la cuarentena ocasionada por la pandemia del Sars covid-19 y fue así como surgieron preguntas:

¿Puede la co-creación aplicarse dentro del entorno virtual? ¿Cuáles son las herramientas más idóneas aplicables al entorno digital? ¿Se puede trabajar colaborativamente con comunidades artesanales o colectivos sociales de manera virtual?

Interrogantes que se acrecentaron a nivel mundial dada la virtualización de la educación y que cuentan con pocas respuestas y escuetas referencias que llevan a pensar que es un camino poco recorrido. Así, la construcción cooperativa dentro de entornos digitales constituye un reto dentro de la planificación de la asignatura, más aún intentar romper 
la idea instaurada de que la co-creación se lleva únicamente en modalidad presencial, un mito que va perdiendo fundamento gracias al aporte que han generado las TIC, cuya contribución ha consistido tanto en la vinculación de actores como en la adaptación de herramientas, programas y almacenamiento en los medios virtuales.

La comunidad universitaria cuenta con varios recursos que apoyan la comunicación entre actores, aportando al proceso de aprendizaje y generando una comunicación sincrónica y asincrónica donde el discente cuenta con gran variedad de herramientas, espacios y recursos al servicio de la construcción colectiva de saberes. Por un lado, herramientas que son parte de los entornos virtuales de aprendizaje (EVA) donde se encuentran recursos organizados de manera didáctica que facilitan la adquisición de conocimiento, respetan las distintas formas de aprender y los diferentes ritmos de apropiación y permiten la retroalimentación grupal e individual entre otros beneficios. Este entorno virtual debe ser un espacio lúdico que permita una interacción social necesaria entre los participantes que genere nexos humanos necesarios para encontrar afinidades y motivar la contribución a la clase. Es necesario ser conscientes de la importancia de crear talleres divertidos, pues el juego es la manera natural de aprender y es a través de esta exploración lúdica que se desarrollan seres más adaptables. Una de las principales características del modelo colaborativo es justamente este componente lúdico que une, integra y promueve la creatividad. Además, permite romper barreras de escolaridad, nivel social, intereses personales entre otros. Como afirma Masferrer (2019. P. 24) "es necesario contar con herramientas accesibles, es decir, inclusivas y fáciles de utilizar por todos", independiente de si se las aplica en espacios virtuales o presenciales.

La modalidad en la que se lleve a cabo la co-creación tiene también características a considerar. La modalidad presencial por su lado tiene el poder de la relación cercana, tan importante en las culturas latinoamericanas y la cosmovisión andina, dónde constituye una de las formas básicas de intercambio personal y social que ha sufrido una brusca adaptación por la situación mundial de distanciamiento generada por la pandemia del Sars covid-19. Es imperante resaltar que a pesar de que la co-creación se desarrolle mayoritariamente en medios virtuales, la aproximación al entorno de la comunidad, sus miembros y su cotidianidad es fundamental así sea llevada en grupos pequeños, pues esto genera confianza, apego y promueve una relación bidireccional de mayor calidez.

La virtualidad en contraparte tiene la ventaja de la atemporalidad, permite romper distancias, fronteras y vincula actores de entornos lejanos. Se pueden aplicar los sentidos de la vista y del oído, pero se pierden el tacto, el olfato y gusto. En el caso de la asignatura se ha suplido estas carencias a través de ejercicios de acercamiento a las prácticas de los artesanos. Ejemplo, realizar el bordado, lo que permite experimentar la actividad desde todos los sentidos, vivenciando los esfuerzos, habilidades y entrega que requiere la tarea. Este tipo de experiencia acerca a los estudiantes a la praxis de los artesanos, poniéndose en sus zapatos y caminando con ellos, de cierta manera, un camino diferente al que acostumbran en su diario vivir y que como objetivo del ejercicio genera reflexión, apertura la visión desde diferentes perspectivas y desarrolla empatía.

Elegir las herramientas adecuadas para cada modalidad es importante, pues constituyen el puente entre los distintos niveles de conocimiento y experiencia, como afirma Masferrer (2019, P.24): 
"Para que un proceso de co-creación discurra de la forma más fluida posible hacen falta herramientas que lo permitan. Con esto me refiero a recursos que equiparen y empoderen los diferentes niveles de conocimiento que un equipo pueda contener. Sobre todo, en equipos multidisciplinares, no todos los miembros tendrán la misma habilidad ni experiencia."

\section{Ventajas y limitaciones de la co-creación en modalidad virtual}

En la mayoría de los proyectos se ha implementado la creencia de que los creativos del equipo (diseñadores, arquitectos, publicistas, entre otros) son los únicos que tienen la capacidad de creación y, se ha puesto sobre ellos la responsabilidad de encontrar ese valor que permita la innovación del proyecto, cuando la creatividad es una característica innata de los seres humanos y todas las personas sin distinción.

La co-creación en la modalidad virtual posee ventajas que provienen desde los actores a partir de los cuales se produce la interrelación y la manera en que es aplicada, es decir: el con qué y cómo se lleva a cabo. Ya que en la interacción aportan tanto los recursos con los que se trabaja, así como la manera en la que se trabaja. Es aquí donde empieza a diferenciarse con los procesos tradicionales o individuales de creación y de dónde surgen sus principales ventajas.

El con qué, hace referencia a los recursos humanos, materiales e inmateriales, tecnológicos, productivos, conocimiento, tangibles e intangibles, tanto de los participantes como del contexto circundante. La principal ventaja que surge de la diversidad de perspectivas, habilidades y experiencias que se tornan en ideas diversas, complementarias y se traducen en diversidad de soluciones al problema planteado.

El cómo, hace referencia a la organización horizontal, sin jerarquías, con capacidad de escucha a todos los implicados en igual medida, con el mismo compromiso e involucramiento, que devenga en un sistema flexible permitiendo que el equipo se acople a circunstancias diversas y cambiantes.

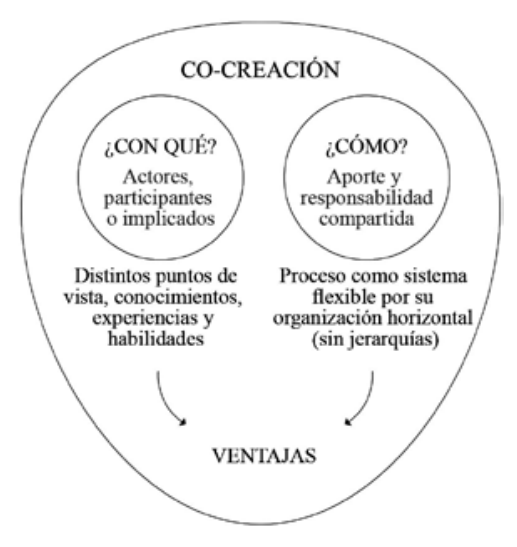

Figura 3. La co-creación como proceso horizontal entre distintos actores.

Nota: El gráfico expone las ventajas de la co-creación que surgen gracias a la participación de distintos implicados y la manera de relacionarlos. 
De estas dos aristas potencializadas desde las TIC se generan ventajas que se exponen a continuación:

- El modelo participativo puede ser aplicado en ámbitos sociales o comerciales a nivel local o global dado el aporte comunicacional de las TIC que rompe distancias y acerca personas de distintas ubicaciones geográficas.

- Se adquiere destrezas tanto desde el desarrollo del proyecto como la adquisición de habilidades desde la práctica mediante entornos virtuales vinculantes y la digitalización que permiten un mayor alcance en menor tiempo y con menor esfuerzo. Ejemplo el levantamiento de información mediante encuestas a través de plataformas. Además, permite una indagación más contextualizada lo que devenga en datos más certeros.

- Adquisición de habilidades en las relaciones humanas, de respeto y trabajo cooperativo en entornos virtuales.

- Capacidad de trabajo sincrónico y asincrónico con la comunidad respetando su propia dinámica.

- Conocimiento del entorno digital desde sus fortalezas y debilidades.

- Capacidad de registro de las sesiones que a su vez permite la repetición de los encuentros grabados.

- Adquisición de experiencias, contactos y vinculación a sectores productivos con mayor apertura dada las facilidades de la virtualidad.

- Libre acceso a la información

Hay que ser conscientes que, dentro de la virtualización de la educación, también existen limitaciones que se aplican a la co-creación en entornos virtuales. Entre ellas tenemos:

- Necesidad de un dispositivo electrónico (teléfono móvil, tableta electrónica, computador) para que la relación pueda darse.

- Conexión a internet, necesarias para la comunicación y acceso a la información.

- Manejo ofimático básico por parte de los participantes.

- Capacidad de sentidos táctiles y olfativos inexistentes o reducidos

- Limitación de interacciones físicas entre los participantes.

- Limitaciones de la virtualidad (tocar el hombro, compartir la comida (sabores), percibir olores del espacio).

- La difícil tarea de coincidir los tiempos de contacto sincrónico entre los participantes del equipo (estudiantes, artesanos, docentes, expertos, etc.) que suelen ser más aptos para conectarse en la noche luego de la jornada laboral.

\section{Algunos métodos para la co-creación}

Durante el último periodo se ha resaltado la importancia de construir colaborativamente por lo que se han generado varios métodos cada uno con su propio enfoque. A continuación, se presentan brevemente algunos de ellos tomados de Giner y Peralt (2013, p.110-111): 
Co-creation innovation ó Co-creación innovación: El término «métodos y técnicas para la co-creation innovation» ha sido utilizado en muchos estudios (Sanders \& Stappers, 2008; Jaworski \& Kohli, 2006; Bitner, Ostrom \& Morgan, 2008; Witell, Kristensson, Gustafsson \& Löfgren, 2011) y lo definen como las distintas actividades donde los consumidores coparticipaban junto con las empresas en el proceso de innovación.

Contextual Design ó diseño contextual: Beyer \& Holzblatt, 1999; Holtzblatt, 2001; Steen et al., 2007 Es una técnica que permite ayudar a los investigadores y diseñadores a través de la observación de personas haciendo tareas en su contexto natural para posteriormente aplicar pragmáticamente sus resultados en el diseño de un sistema o un producto. Educación On-line

Co-Design ó co-diseño: Sanders, 2000, 2002; AndersonConnell, Ulrich \& Brannon, 2002; Sanders \& Stappers 2008. Consiste en una actividad de cocreación colaborativa en el desarrollo de producto y la fase de prototipo, donde los profesionales del diseño y de la investigación potencian, estimulan y guían a los clientes para lograr definir, configurar, un producto o modificarlo en función de sus necesidades.

Participatory Design ó diseño participativo: Schuler \& Namioka, 1993; Kensing \& Blomberg, 1998; Spinuzzi, 2005 Se involucra activamente a todas las partes interesadas (por ejemplo, empleados, socios, clientes, ciudadanos, usuarios finales) en el proceso de diseño con el fin de ayudar a asegurar que el producto diseñado se ajuste a sus necesidades y sea perfectamente utilizable.

\section{El diseño participativo aplicado al Taller de Diseño}

El diseño participativo surge del método escandinavo dónde se busca la practicidad de los objetos, naciendo como un movimiento de la democracia laboral donde se toma en cuenta la perspectiva del trabajador como implicado conocedor del proceso. Posteriormente, en Norteamérica, se transforma como afirma Gross (2019, P.7), con "énfasis en la participación de los usuarios, más que en el componente colaborativo o en la voluntad de empoderamiento y emancipación", vinculado al concepto del diseño centrado en las necesidades del usuario. Las características del trabajo participativo para Barkley, Cross, \& Howell (2007, PP.18-19) son:

1. El diseño intencional: consiste en diseñar actividades de aprendizaje en base a una serie o estructuración.

2. La colaboración: es el aporte equitativo de cada miembro del grupo.

3. Enseñanza significativa: cumple objetivos de aprendizaje útiles para el estudiante dónde sea responsable de su auto aprendizaje de manera activa.

En la asignatura de Taller de Diseño correspondiente a la sección formativa de la carrera de Diseño de Productos de la PUCE, el Diseño Participativo se aplica como una metodo- 
logía que permite unificar los valores y las metas que cada grupo de participantes posee. Por una parte, la academia desde su rol, socialmente aceptado, de legitimadora y productora de conocimiento (a pesar de no ser la única), posee ciertos valores y metas que distan de los valores y metas que tiene la comunidad, así a través de esta metodología y después de un largo camino de exponer y dialogar, se logra unir las metas en un fin compartido sobre el cual todos aportan y construyen.

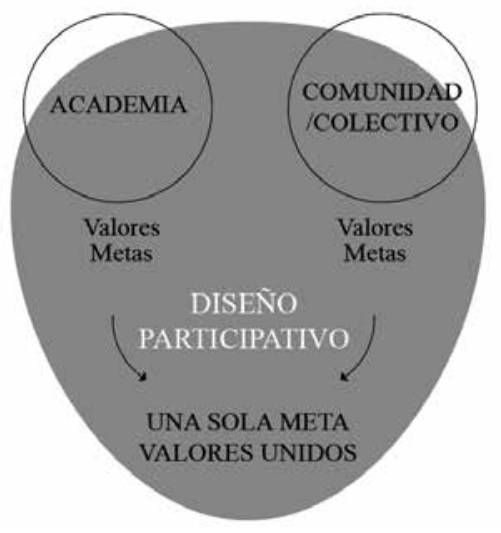

Figura 4. Unión de valores y metas.

Nota: El gráfico demuestra como la metodología une los valores y metas de los diferentes actores.

Proceso que debe darse de manera continua y en periodos de tiempo prolongados que permitan a todos los actores relacionarse e involucrarse en lo cotidiano, pues la realidad no puede ser contada, es más fructífera cuando se experimenta dentro de su fluir natural. En este aspecto Robertson \& Simonsen (2012), como se citó en Gros (2019, p. 8) afirman:

"El diseño participativo no debe entenderse como una mera intervención de los implicados. Participar, desde esta perspectiva implica investigar, reflexionar, comprender, proponer, desarrollar y apoyar mutuamente los procesos de aprendizaje entre los participantes a lo largo de todo el proceso"

A partir de entender que el proceso debe darse en todo momento y a todo nivel, en la cátedra de Taller se desarrolla un cronograma adaptado a los tiempos académicos, donde a pesar de no ser el más apto por su duración de 16 semanas, logra desarrollar todas las fases de un proyecto de diseño de manera cooperativa. Bajo esta premisa se manejan acercamientos entre los actores que van entre una a dos reuniones semanales, y durante ese tiempo se van estableciendo convenios entre la comunidad y la universidad mediante sus facultades, carreras, eventos, proyectos o programas (como la Feria de Economía Popular y Solidaria), buscando nuevas alianzas y abriendo nuevos medios de exposición de los productos para llegar a los consumidores. Es decir, se busca el mantener la vinculación 
entre la academia y la comunidad como nuevas estrategias de instaurar una universidad más ligada a la realidad social donde a partir de una educación más abierta e inclusiva se logre una transformación social y se entrelacen saberes de distintas fuentes.

Esta adaptación temporal es consensuada en base a los tiempos, tanto de la comunidad cómo del semestre académico, por lo que se sugiere empezar su planificación antes de que el ciclo de inicio, pues la organización dentro de la comunidad como contraparte tiene su propia dinámica (unas veces jerárquica, otras veces de carácter organizativo) y siempre con el ideal de que el proyecto construido durante los cuatro meses que dura la cátedra, sea el inicio de un largo trabajo conjunto y se extienda a futuros semestres en distintas asignaturas, con los mismos alumnos de ser posible e ir sumando nuevos estudiantes al proceso, para tener un intercambio sostenido que siente bases sólidas de confianza e impacto.

Cuando el ciclo inicia, se debe empezar por tener organizadas las semanas de trabajo ya que cada una es un paso adelante para lograr la meta establecida. Esto no constituye una camisa de fuerza, pues es imperante que se tome en cuenta la dinámica del grupo para que el proceso avance de manera respetuosa con los participantes y los ritmos de las partes implicadas. Es importante tener diseñadas las herramientas que se van a aplicar, teniendo los objetivos definidos de manera que se aproveche el tiempo junto a la comunidad pues como se expuso previamente constituye uno de los limitantes de la virtualidad. Adicionalmente, se debe permitir que el estudiante asuma el rol de organizador, creador de herramientas e indagador. Desde esta perspectiva se presentan técnicas y herramientas útiles para desarrollar las mingas virtuales.

\section{Herramientas para la Minga Virtual}

Para llevar con éxito el trabajo colaborativo entre actores de diferentes orígenes, es imperante que se cuente con herramientas, técnicas y ejercicios que permitan la interacción lúdica de los implicados a través de la modalidad virtual. Es importante tomar en cuenta que dichas herramientas deben constituir un punto de unión, de convergencia de conocimientos, saberes y experiencias. Por ello, los espacios virtuales en los que se apliquen estas herramientas deben ser espacios de confianza, cálidos y divertidos que promuevan sinapsis.

En la asignatura de Taller de Diseño Participativo se aplicaron técnicas y herramientas que se potenciaron a través de las TIC y que junto a la comunidad de bordadoras Sara Sisa de Llano Grande, se pudo obtener datos para la generación del proyecto de diseño. A continuación, se presenta un recorrido por recursos que fueron útiles para la minga virtual con imágenes correspondientes al semestre agosto a diciembre del 2020.

\section{Ejercicios apoyados en medios digitales}

Al inicio de cada sesión, se realizan actividades rompe hielos que permiten a los participantes conocerse y empezar a empatizar. Entre los recursos digitales que se utiliza están: imágenes para describirse si fuese una comida, fotografías de los juguetes favoritos de la infancia, o se interactúa entre cuadrantes de Zoom para dar y recibir objetos como una 
tijera donde tanto la persona que entrega como la que recibe deben pensar en el otro participante para realizar la acción. Este tipo de ejercicio nos acerca a los lugares de procedencia, a la cultura gastronómica, a los hobbies, entre otros que nos permiten conocer a las personas, crear lazos, descubrir gustos en común y generar simpatía.

\section{Rompecabezas de un cuadro}

Esta actividad promueve la construcción cooperativa con el objetivo de diferenciar el trabajo en el que se unen partes divididas versus la tarea que fue creada por todos los participantes en base a discusión y reflexión grupal. Los participantes son quienes deciden los recursos, elementos y demás pasos durante el proceso, lo que permite adquirir destrezas de planificación.
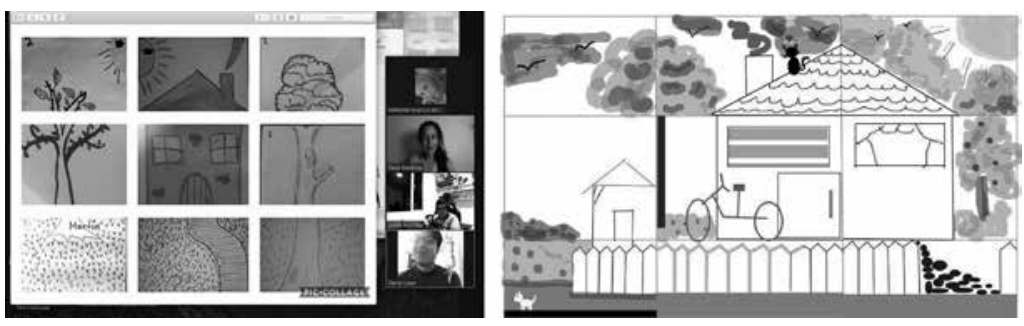

Figura 5. Cuadros de co-creación. Nota: La imagen muestra el ejercicio realizado a mano con armado por medios ofimáticos, como también el ejercicio realizado de manera digital.

Este tipo de ejercicios deja varias enseñanzas a nivel de planificación de actividades y coordinación entre miembros del equipo, por lo que se sugiere el análisis al finalizar la exposición para que sean los mismos participantes quienes realicen la reflexión en la acción. El ejercicio duró 30 minutos de los cuales 17 minutos se emplearon en la planificación, 10 minutos fueron necesarios para realizar la tarea y 3 minutos para socializarla. A continuación, un resumen de lo aprendido en la dinámica:

Llegar a acuerdos: por ejemplo, en la orientación del formato que se había especificado como A4, instrumentos a utilizar para que se vean las líneas con el mismo grosor y formen una sola composición, entre otros.

Dialogar: Para definir el contenido por cuadrantes, elementos a dibujar en la composición (en los cuadrantes inferiores va el piso, el camino, etc.), especificar que va a contener cada cuadrante (y así no tener dos soles).

Hacerse escuchar y saber escuchar: pues todos tienen ideas igual de importantes y deben de aportar a la construcción de la consigna.

División de tareas y responsabilidades: definir que cuadrante utilizaba cada alumno y si entre cuadrantes se debía acoplar elementos de manera conjunta (árbol, casa, camino). Cumplir tiempos y cronogramas: planificar el tiempo de acuerdo con las actividades, la 
secuencia del trabajo (si todos bocetan al mismo tiempo los elementos no iban a coincidir el momento de juntar los cuadrantes).

No cargarse el trabajo de otros participantes, aportar si es necesario: cada estudiante tenía asignada su responsabilidad de trabajo (cuadrante), lo que debía contener y cómo debía ser su vinculación con los cuadrantes cercanos.

Acoplarse y ceder: pues la construcción colectiva implica diálogo y discusión, saber hasta donde es el rol de un actor para que inicie el de otro.

Este tipo de ejercicio surge con el propósito de romper la instaurada idea de que un trabajo en grupo es la realización de parte del proyecto para luego ser unido con las otras partes sin que haya total concordancia entre los aportes independientes, y aún peor, sin que haya discusión, reflexión y construcción colectiva que constituye la meta del ejercicio.

\section{Encuesta}

La encuesta es uno de los recursos que se potencia con las TIC gracias a plataformas como google forms, surveymonkey, survio, zohosurvey, e-encuesta, eval\&go, entre otras. Se pueden construir formularios con preguntas de distintos tipos capaces de difundirse por varios medios digitales mediante un enlace, entre sus ventajas está la facilidad de la recolección de datos, la generación de resultados al instante y el costo. La distribución dentro de la audiencia correcta está definida por el investigador quien enfoca la herramienta el momento de enviar el enlace. En el ejercicio académico este recurso fue útil en dos momentos: 1) cuando las doce mujeres de la comunidad seleccionan los tipos de objetos a desarrollar y 2) para elegir el concepto final, de las tres alternativas que se co-crearon a partir de sus relatos.

\section{Entrevistas}

La entrevista es un recurso útil que se puede llevar sin problemas en la virtualidad. Existen entrevistas atemporales como las encontradas en la web que tienen la ventaja de la atemporalidad, pero no permiten intercambio bidireccional. También están las entrevistas realizadas en tiempo real, que se pueden llevar a cabo por aplicaciones como Facetime, WhatsApp en video llamada, Zoom, Meet, entre otros, dónde los alumnos logran interactuar y levantar información faltante a través de un cuestionario.

\section{Etnografía}

La etnografía se adapta a los medios digitales, ya que no implica actividad ni intercambio temporal por lo que recursos como grabaciones de videos, visitas digitales guiadas, documentales, canales de las comunidades, entre otros, pueden ayudar a realizarla desde la virtualidad permitiendo obtener información valiosa para el proyecto. A través de la etnografía los estudiantes pudieron acercarse al entorno del Llano Grande en medio de la cuarentena, encontraron personajes de influencia, conocieron el entorno natural de sus plantas y frutos representativos, descubrieron las tribus urbanas que cohabitan, se instruyeron sobre los tipos de puntadas del bordado que se realizan solo en esta comuna, entre otra información relevante sobre la zona. 


\section{Observación Participante}

Esta herramienta es la suma de la observación más la acción, permite al investigador tener un acercamiento mayor al contexto e involucrarse directamente con los actores ya que se trata de realizar las tareas junto a los sujetos observados. Se da en tiempos prolongados donde puede encontrar información de la dinámica natural del medio y descubrir los roles principales o que no se exponen dentro de la estructura de la comuna. Como define Lorda (2011, p.97) "la observación participante o participativa alude a la inmersión de un observador en una comunidad, a una cultura determinada, en donde a lo largo de un tiempo permanece en dicho sitio con el objeto de observar a los individuos en un recorte de su vida cotidiana." En los medios digitales la observación participante es limitada, pero puede aplicarse a través de plataformas de videoconferencias como Zoom o Meet que son medios útiles para poder observar, participar, intervenir en la actividad a distancia. Los estudiantes realizaron la actividad de bordar para poder experimentar el proceso en todas sus facetas y como resultado obtuvimos nuevas opiniones sobre el bordado que, pasó de ser una decoración para entenderse como un medio de empoderamiento, de la lucha colectiva de las mujeres de Sara Sisa por la independencia económica frente a un sistema patriarcal.

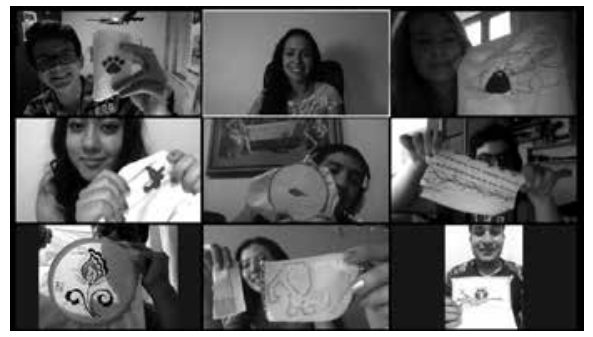

Figura 6. Estudiantes experimentan el bordado con diferentes puntadas. Nota: El ejercicio permite entender todo el proceso, acercarse a los materiales y herramientas.

\section{Storytelling ó narración y pirámide de Freytag}

La narración también conocida como storytelling, es una herramienta que permite crear una historia real, imaginaria o mixta a partir de situaciones, objetos o creencias que forman parte de la comunidad. La cultura oral es uno de los medios por los que más se mantienen las costumbres, resultando un ejercicio muy cómodo para los participantes logrando impregnar de emocionalidad e información sensible al relato. Es a través de la pirámide de Freytag, creada por el novelista alemán Gustav Freytag, que la narración se estructura encontrando los puntos principales para, después de pasar por un proceso de escritura, gráfica y de representación objetual, da como resultado el concepto del proyecto.

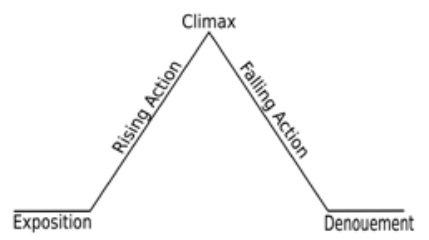

Figura 7. Pirámide de Freytag utilizada para estructurar el relato de las bordadoras.

Fuente: Imagen tomada de: http:// cervantesobservatorio.fas.harvard. edu/en/node/398 


\section{Talleres de bocetación y maquetación}

En el desarrollo del producto son de gran utilidad los talleres de bocetación y maquetación, pues junto a la comunidad se configuran las ideas que permiten visualizar las alternativas de solución que por la metodología y la diversidad de actores resultan ser variadas. Es importante recordar que los materiales solicitados para la experimentación deben ser simples, económicos y posibles de encontrar en los hogares de todos los participantes.

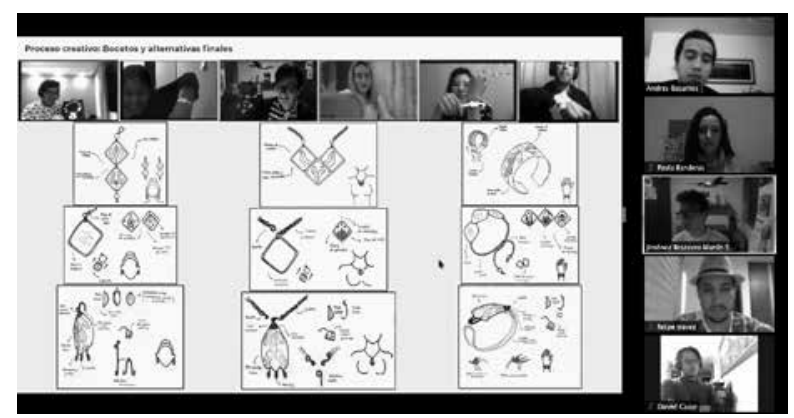

Figura 8. Taller de bocetación y maquetación rápida. Nota: En la imagen se puede ver a diferentes actores: estudiantes, bordadoras, profesionales y docentes. Se definen detalles del producto y se conoce las diferentes percepciones.

\section{Mapa de empatía}

Diseñado por el diseñador Dave Gray, nace como una herramienta mediante la cual se potencia la visión estratégica de los clientes. Es así nacen preguntas relacionadas a qué ve, siente, dice o hace o a quien escucha el cliente, pudiendo definir si sus acciones concuerdan con su discurso. Ello permite conocer sus motivaciones y frustraciones para encontrar puntos sobre los cuales trabajar.
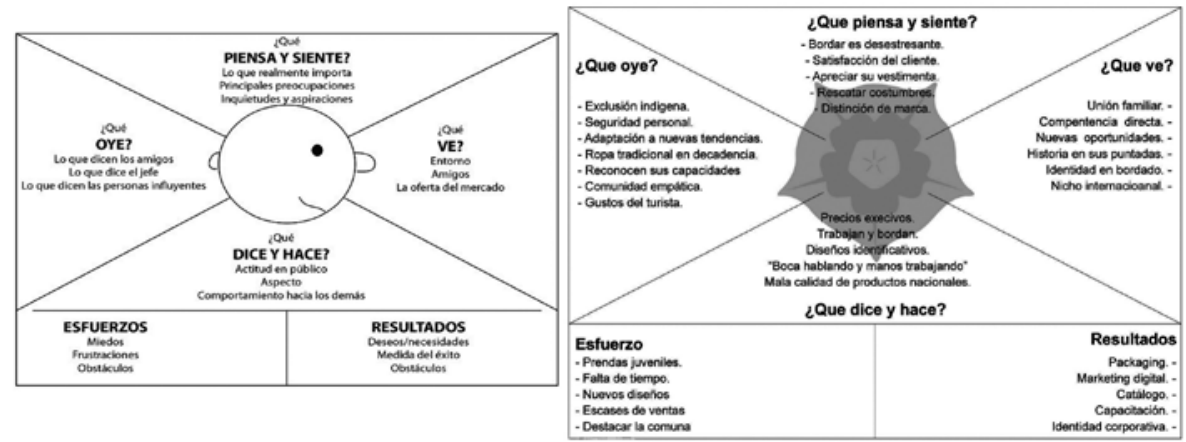

Figura 9. Mapa de empatía. Fuente: plantilla tomada de https://www.ideo.com search?q=empathy\%20 map. Ejercicio aplicado por estudiantes del semestre agosto a diciembre del 2020. 


\section{Boards/paneles: moodboard/panel de tendencia, socialboard/panel social, conceptboard} /panel de concepto, Brandboard/panel de marca, entre otros

Los boards o paneles visuales son herramientas creativas que permiten una visualización de fotografías, palabras o frases, texturas, patrones, colores, tipologías de objetos entre otros, con el fin de inspirar. Estas imágenes pueden ser realizadas en físicos (recortes de revista o páginas impresas), así como digitalmente en varias plataformas (Canvas, Pinterest, Niice), por lo que son un excelente recurso en los ejercicios de la minga virtual.

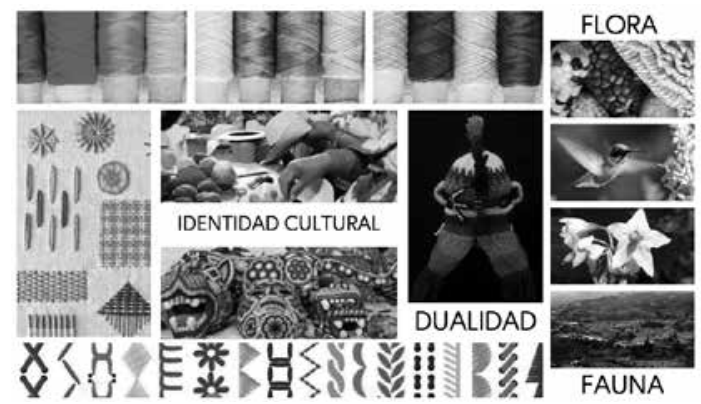

Figura 10. Moodboard sobre Sara Sisa. Fuente: Identidad de Sara Sisa, realizado por estudiantes del semestre agosto a diciembre del 2020.

\section{Matriz de viaje en el tiempo}

La matriz de viaje en el tiempo es un recurso que permite regresar al pasado (época neandertal) para conocer cómo se realizaba la actividad o se solucionaba el problema. En el presente nos ayuda a describirla y, hacia el futuro, nos permite imaginarnos soluciones sin límites de factibilidad lo que devenga en ideas innovadoras para luego asentarlas a la realidad. Es un recurso que se puede desarrollar cooperativamente de manera virtual sincrónica o asincrónica.

\begin{tabular}{|c|c|c|c|}
\hline & 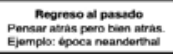 & $\begin{array}{l}\text { En el prosente } \\
\text { Ponere di ta acción o problema }\end{array}$ & 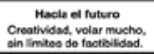 \\
\hline 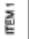 & & & \\
\hline ᄅe & & & \\
\hline ב己 & & & \\
\hline & & & \\
\hline
\end{tabular}

Figura 11. Matriz de viaje en el tiempo.

Fuente: Matriz realizada por autor en base al curso De la educación Popular al diseño participativo: Metodologías para la co-creación. Trávez L. y Fuentes D., 2019. 


\section{Diagrama o mapa de afinidad}

El diagrama de afinidad permite clasificar gran cantidad de conceptos, agruparlos y relacionarlos. Se trata de escribir cada concepto en un Post it, que pegados en un espacio amplio donde todos puedan visualizarlos se proceda a organizarlos por asociación (http://www.sidar.org/). Algunas sugerencias que apoyan al correcto funcionamiento del recurso son: escribir con letra imprenta usando mayúsculas, usar una o pocas palabras en cada post it, usar el mayor tamaño de letra posible y usar un color de marcador que contraste con el color del fondo. En este sentido existen plataformas virtuales y gratuitas como lucid.app que recrea el recurso del post it en un tablero, permitiendo todas funciones necesarias para el ejercicio.

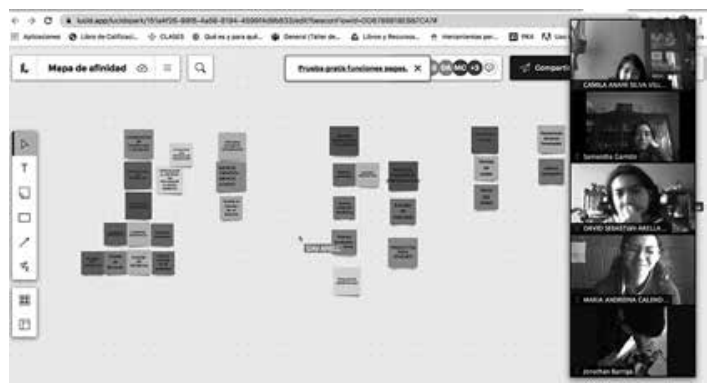

Figura 12. Mapa de afinidad.

Nota: la imagen permite ver la recreación de post it en aplicaciones digitales.

\section{Sistema de acuerdos}

Esta herramienta es un excelente recurso para definir si las ideas de los participantes son certezas, suposiciones o dudas. Consiste en votar mediante tres tipos de tarjetas sobre una idea presentada. Las tarjetas con colores permiten:

a. Tarjeta de color rojo: significa estar en desacuerdo con la idea,

b. tarjeta de color amarillo: significa punto medio, es pensar "puedo vivir con ello",

c. tarjeta de color azul, quiere decir que se apoya la idea por lo está de acuerdo.

Si entre todos los participantes se obtiene más del 33\% de tarjetas rojas, la idea se anota como duda y debe definirse como certeza mediante las herramientas de investigación que se considere. Si en algún momento gana el rojo, una de las personas que votó en desacuerdo debe proponer una idea. De esta forma se llena el cuadro de certezas, suposiciones y dudas para trabajar el proyecto sobre las certezas que se establezcan.

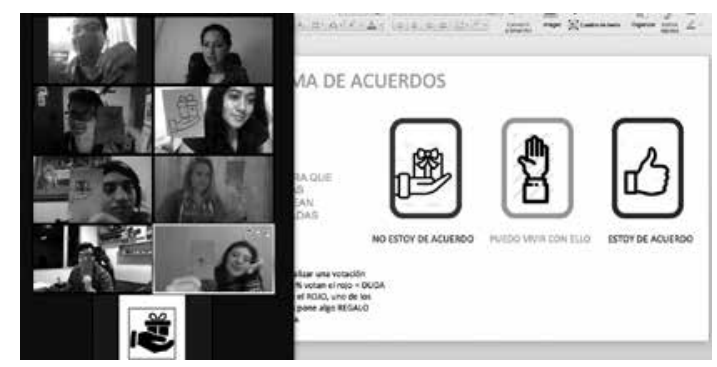

Figura 13. Tarjetas del sistema de acuerdos. Nota: estudiantes dando su opinión utilizando las tarjetas del sistema de acuerdos. 


\section{Votación Puntocracia}

Es una estrategia de votación que considera la opinión de todos los participantes en la tarea de decidir sobre una alternativa propuesta. También denominada multi-votación, votación de stickers, punto democracia, entre otros, permite a los participantes votar por más de una opción. Es así como del número de opciones a elegir se divide para 2 y se suma 1. Ejemplo, si tenemos 6 alternativas: $(6 / 2)+1=4$, así los participantes tienen 4 puntos, stickers, rayas o lo que fuere para señalar sobre su alternativa de preferencia. La alternativa que más votaciones tenga gana.

\section{Un día en la vida de...}

Herramienta interesante al momento de observar y encontrar insights. En la virtualidad nos ha servido de manera atemporal donde las participantes de la comunidad filman una actividad dentro de su cotidianidad (cocinar, bordar, bailar, tocar música, cosechar, etc.) y los envían por medios digitales para que subidos en la wiki del EVA se pueda analizar por los demás participantes. Por otro lado, de manera temporal mediante video llamadas que además de la observación han permitido la acción, permitiendo que se aplique además la observación participante en pocos casos ya que demanda de mayores recursos como tiempo de dedicación a la tarea, coincidir en horarios, así como megas de internet para mantener la conexión.

Evaluaciones: diagnóstica, formativa, sumativa.

\section{Coevaluación, heteroevaluación, autoevaluación.}

Las evaluaciones se realizan durante varias fases del taller. Al empezar se realiza una evaluación diagnóstica para conocer el grado de conocimiento del grupo de estudiantes. En el transcurso de las clases y a través de las tertulias y ejercicios se realiza una evaluación formativa y como ejercicio académico se aplica la evaluación sumativa. Dentro de las entregas de trabajos se promueve la coevaluación pues es un ejercicio horizontal en el que todos los actores cuentan con la misma voz, voto y el compromiso de aportar colaborativamente. La heteroevaluación se aplica en los cierres de las fases de investigación, desarrollo del proyecto y validación donde se cuenta con la presencia de profesionales y docentes invitados que acompañan durante todo el proceso. La autoevaluación se realiza mediante la herramienta de encuesta de la plataforma Google forms, para definir el nivel de conocimiento adquirido durante el semestre en relación con los resultados de aprendizaje de la materia.

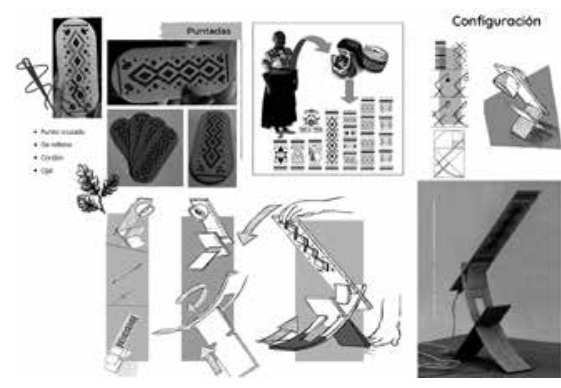

Figura 14. Evaluaciones.

Nota: La imagen muestra distintos procesos de evaluación en las fases de desarrollo del producto. Proyecto del estudiante Edison Pazmiño en co-creación con Sara Sisa. 
Los recursos compartidos constituyen un apoyo fundamental para lograr el compromiso de los actores e incentivarlos a participar de las reuniones periódicas. Como se puede apreciar en los ejemplos, las herramientas pueden aplicarse en varias etapas del proyecto logrando alcanzar tres puntos importantes a considerarse en una investigación participativa. Como exponen Bustamante, Brendel, Degbelo \& Kray (2018, p. 36):

Respondiendo sobre los aspectos importantes antes de iniciar una investigación participativa, se debe considerar principalmente generar un ambiente seguro o de confianza, lo cual corresponde a brindar apertura ante lo que las personas que participan lleguen a aportar, además de crear un ambiente de confidencialidad para expresar toda clase de comentarios. Seguido a esto, se debe establecer los niveles de participación, es decir, que se debe exponer con claridad lo que se espera de cada uno de los participantes en cada etapa del proceso. Y tercero se debe definir el impacto que se desea obtener, lo cual tiene dos objetivos de su realización: buscar entender la realidad social y realizar cambios para mejorar esta realidad, comprendiendo qué implica hacerlo.

El siguiente mapa presenta el camino durante el Taller de Diseño Participativo, donde los actores involucrados en el proyecto se conectan mediante la metodología del diseño participativo para, a partir de la co-creación como proceso horizontal y respetuoso, unir los valores y metas de cada grupo de participantes convirtiéndolas en un solo fin para, mediante el proyecto cargado con el valor generar impacto en todos los involucrados.

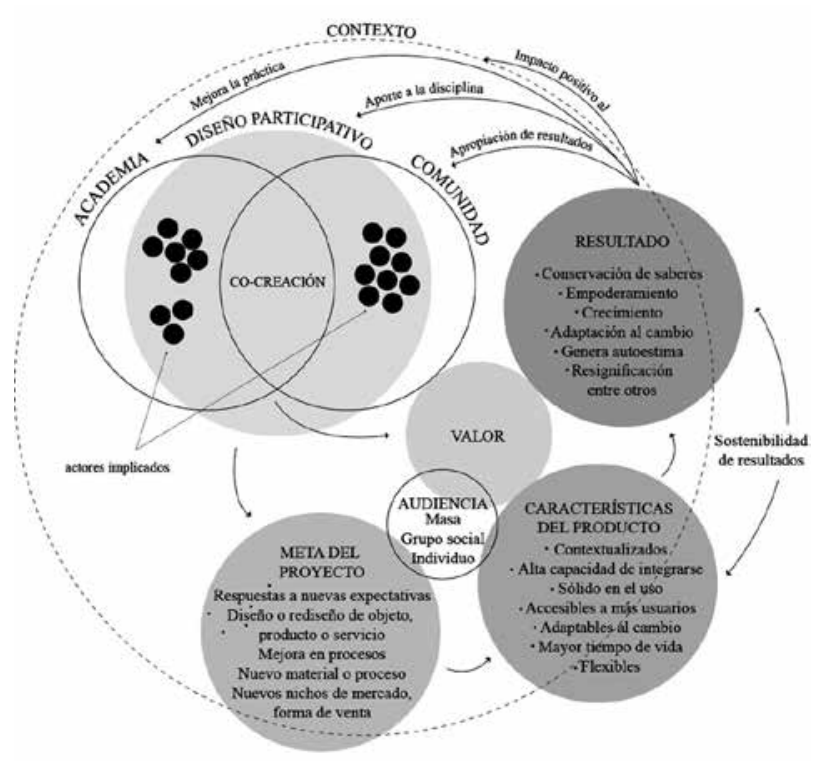

Figura 15. Actores involucrados en el proceso de co-creación dentro de la metodología de Diseño Participativo. Nota: El gráfico demuestra los niveles de impacto del proceso participativo. 
Esta metodología busca lograr una transformación profunda de las situaciones, necesidades y problemáticas, porque se las observa, estudia e investiga tomando en cuenta varias perspectivas, que a partir de la co-creación generan una diversidad de soluciones que son adaptables y flexibles. Como ventajas de este proceso se obtiene un diseño que sea apto para mayor número de personas y que los usuarios se identifiquen, se apropien del resultado obtenido y por consiguiente que se alcance su implementación. Este último punto ha sido difícil de lograr en los procesos llevados a cabo en el contexto ecuatoriano por empresas público y privadas, dado que las metodologías aplicadas no han permitido el aporte de los implicados. Motivo por el cual se encuentra mucha desconfianza de los artesanos y comunidades, ya que sienten que los acercamientos de las instituciones (sean académicas, privadas o gubernamentales) se propician para obtener beneficio unilateral.

Es importante considerar que el fin del diseño participativo no es un producto entendido como objeto, servicio, mejora en el proceso, entre otros, la finalidad es el impacto que éste pueda causar al medio en que se lo desarrolla, comercializa y utiliza.

Es común en proyectos de apoyo a comunidades, el obtener resultados que solo sirven para cerrar el ciclo de los talleres o proyectos privados y que se queden solo en buenas intenciones, cuando el sentido de la investigación y el verdadero trabajo se realiza para verlo implementado y a futuro cosechar los frutos que son la meta verdadera. Como explican Durall \& Leinonen (2014), "una construcción social y los resultados del diseño como algo que va a tener un impacto real en la realidad socialmente construida que la gente vive, requiere responsabilidad y rendición de cuentas por parte de los diseñadores y de las personas que participan en el diseño" (como se citó en Gross, 2019)

El éxito de este modelo se debe en gran medida a como afirma Gross $(2019$, p.63): "la reflexión en la acción que revierte en todos los participantes en forma de un mayor conocimiento y comprensión sobre el contexto, las prácticas que tienen lugar y los artefactos diseñados". Es así como, el crecimiento y aprendizaje que surgen de esta experiencia se da de manera colectiva como resultado de la actividad realizada de igual forma. Esta construcción permite que los participantes sientan que es un verdadero aporte para su vida cotidiana y esto hace que se aplique y replique.

\section{Conclusiones}

La co-creación toma su verdadera fuerza cuando es aplicada en la academia como una metodología de cambio, de transición de la educación desde la jerarquía y el poder hacia la horizontalidad y la construcción de conocimiento desde la cooperación y diversidad de saberes. Tiene su principal atributo como transformadora del sistema académico, legitimador y fiscalizador del conocimiento, y debe darse desde cada institución, así como desde cada aula de clase. Por ello, el ejercicio de Taller de Diseño Participativo vinculado a las comunidades del país pretende aportar a construir una Universidad abierta, permeable, solidaria, inclusiva, empática y vinculada con la gente y para la gente.

El sistema educativo vive una transformación profunda en la cual es imperante encontrar estrategias de enseñanza aprendizaje que permitan diferenciarse y adaptarse a las nuevas 
necesidades del mundo post pandémico, donde la virtualidad es una realidad que ha llegado para quedarse. Metodologías inclusivas apoyadas en las TIC se vuelve una poderosa arma para enfrentar la compleja tarea de acercarse al estudiante y vincularlo a la realidad profesional.

La co-creación aplicada en la academia sea como proceso, estrategia y/o como metodología de trabajo, puede desarrollarse en la presencialidad y en la virtualidad. En ambos casos los espacios y las herramientas deben ser lúdicas y estar planificadas y adaptadas a cada modalidad, tomando en cuenta que sean inclusivas y tiendan puentes entre los participantes.

El acercamiento del discente a la realidad del país crea nexos con artesanos y profesionales, desarrolla criterios de elección de herramientas para su aplicación dependiendo la naturaleza del proyecto, crea en el estudiante confianza para enfrentarse al ejercicio profesional, rompe miedos y frustraciones frente a lo desconocido, a lo distinto y al cambio. Desarrolla capacidad de aceptación, compresión y empatía, se aprende a planificar respetando tiempos y maneras de fluir de cada contexto y sobre todo enfrenta los proyectos desde una posición igualitaria donde aporta desde sus conocimientos y valida los distintos saberes. El desarrollo de métodos de co-creación a nivel virtual son factibles gracias al compromiso, entrega, compañerismo y aporte tanto cognitivo como práctico de todos los participantes.

\section{Notas}

${ }^{1}$ Sociedad se refiere a un grupo humano que tiene por base el individuo para establecer relaciones abiertas, mientras que una comunidad es un tipo de vínculo en el que lo medular es la sangre, la cultura y creencia común, también el apego afectivo a un valor. (Castro, 2019, p. 147)

\section{Bibliografía}

Aubert, A., Flecha, A., García, C., Flecha, R. y Racionero, S. (2008). Aprendizaje Dialógico en la Sociedad de la información. Barcelona: Hipatia Editorial.

Barkley, E., Cross, D. \& Howell, C. (2007). Técnicas de aprendizaje colaborativo. Madrid: Morta

Borja, R. (2012). Enciclopedia de la Política. México D.F. Fondo de Cultura Económica.

Bruna Castro, Carolina. (2019). Sobre la relación entre individualidad y colectividad. Notas a el hechicero de la tribu. Mario Vargas Llosa y el liberalism en America Latina de Atilio Borón. Atenea (Concepción), (520), 147-154. https://dx.doi.org/10.4067/S071804622019000200147

Cruz, Miguel. (2018). Cosmovisión Andina e Interculturalidad: Una mirada al desarrollo sostenible desde el Sumak Kawsay. Revista Chakiñan de Ciencias Sociales y Humanidades, 
(5), 119-132. Recuperado en 13 de marzo de 2021, de http://scielo.senescyt.gob.ec/scielo. php?script=sci_arttext\&pid=S2550-67222018000100119\&lng=es\&tlng=es.

Gros, Begoña. (2019). La investigación sobre el diseño participativo de entornos digitales de aprendizaje. University of Barcelona.

Hall, Budd L. (1983). Investigación participativa, Conocimiento Populary Poder: una reflexión personal. México: Biblioteca Digital CREFAL.

Lordas, M. (2011). Aportes para la investigación geográfica a partir de la observación participante y de entrevistas. M Lorda - Huellas, 2011 - biblioteca.unlpam.edu.ar

Masferrer, Alejandro. (2019). Diseño de procesos creativos: Metodología para idear y co-crear en equipo. Barcelona: Gustavo Gili.

Real Academia Española. (s.f.). Minga. En Diccionario de la lengua española. Recuperado en 12 de abril de 2021, de https://dle.rae.es/minga

Real Academia Española. (s.f.). Co. En Diccionario de la lengua española. Recuperado en 12 de abril de 2021, de https://dle.rae.es/co-

Real Academia Española. (s.f.). Crear. En Diccionario de la lengua española. Recuperado en 12 de abril de 2021, de https://dle.rae.es/creaci\%C3\%B3n?m=form

Ribes Giner, G., \& Peralt, A. (2014). Methods and techniques facilitators of co-creation innovation in master programs for postgraduate market. Intangible Capital, 10(1), 101 124. doi:http://dx.doi.org/10.3926/ic.443

Ron, José. (1997). Sobre el concepto de cultura. Quito: IADAP.

Sánchez Hernández, Arturo José. (2005). Análisis filosófico del concepto valor. Humanidades Médicas, 5(2) Recuperado en 01 de abril de 2021, de http://scielo.sld.cu/scielo. php?script=sci_arttext\&pid=S1727-81202005000200009\&lng=es\&tlng=es.

Wilches, D. (2019, 13 diciembre). Aportes de la co-creación para la innovación y las relaciones con clientes. http://www.scielo.org.co/.http://doi.org/10.14349/sumneg/2020.V11.N24.A9

\begin{abstract}
The year 2020 faced the SARS-COV2 pandemic, which brought with it the virtualization of education worldwide and with it new challenges in teaching-learning strategies. This leap was a great step for education where the success of the process responds to the commitment of the actors to the chair and the modality. An example of this process has been the Participatory Design Workshop, a subject that belongs to the formative section of the redesign of the PUCE Product Career and has the particularity of developing co-creation methods with vulnerable communities that have little access to design. and the benefits it provides. In the second semester of 2020 this subject was taught for the first time and developed in collaboration with the Sara Sisa community. This group of indigenous women from Llano Grande is recovering their customs through embroidery and have found in this technique a source of economic income, personal satisfaction, empowerment and collective development.

This article presents tools that allow the academy to approach the local reality through the virtual minga as a cooperative process for the construction of projects that take into account differences as strengths for the construction of innovative products with value. It
\end{abstract}


should be noted that the institution, with its background of quality training, has developed various means of virtual communication between teachers and students, which has made it possible to train trained professionals, empathic and committed to society, from various angles, demonstrating that the processes of cooperation Creation can be successfully supported by the new information and communication technologies.

Keywords: Participatory design - virtual education - ICT - collaborative projects - cocreation.

Resumo: O ano de 2020 enfrentou a pandemia SARS-COV2, que trouxe consigo a virtualização da educação em todo o mundo e com ela novos desafios nas estratégias de ensino-aprendizagem. Esse salto foi um grande passo para a educação onde o sucesso do processo responde ao comprometimento dos atores com a cadeira e a modalidade. Um exemplo desse processo foi a Oficina Participativa de Design, tema que pertence à seção formativa do redesenho da Carreira de Produtos PUCE e tem a particularidade de desenvolver métodos de cocriação com comunidades vulneráveis e com pouco acesso ao design. benefícios que oferece. No segundo semestre de 2020 esta disciplina foi ministrada pela primeira vez e desenvolvida em colaboração com a comunidade Sara Sisa. Este grupo de mulheres indígenas de Llano Grande está resgatando seus costumes através do bordado e encontraram nesta técnica uma fonte de renda econômica, satisfação pessoal, empoderamento e desenvolvimento coletivo.

Este artigo apresenta ferramentas que permitem à academia se aproximar da realidade local por meio da minga virtual como um processo cooperativo para a construção de projetos que levam em conta as diferenças como pontos fortes para a construção de produtos inovadores com valor. Ressalte-se que a instituição com sua trajetória de formação de qualidade tem desenvolvido diversos meios de comunicação virtual entre docentes e discentes, o que tem possibilitado formar profissionais capacitados, empáticos e comprometidos com a sociedade, sob diversos ângulos, demonstrando que os processos de cooperação A criação pode ser apoiada com sucesso pelas novas tecnologias de informação e comunicação.

Palavras-chave: Design participativo - educação virtual -TIC -projetos colaborativos cocriação.

[Las traducciones de los abstracts fueron supervisadas por el autor de cada artículo] 